\title{
A COUPLING TECHNIQUE FOR STOCHASTIC COMPARISON OF FUNCTIONS OF MARKOV PROCESSES
}

ENSEEIHT, 2 rue Camichel, 31071 Toulouse, France.

doisy@len7.enseeiht.fr

\begin{abstract}
The aim of this work is to obtain explicit conditions (i.e., conditions on the transition rates) for the stochastic comparison of Markov Processes. A general coupling technique is used to obtain necessary and sufficient conditions for the construction of a coupling Markov Process which stays in a fixed set $K$ for all times and with given marginal processes. The strong stochastic comparison - or, more generally, the stochastic comparison through states functions - appears as a particular case. An example in the Reliability Theory is developed and proves the efficiency of the method. Systems with multiple component types and redundant units are stochastically compared directly or through particular functions.
\end{abstract}

Keywords: Stochastic Comparison, Coupling, Markovian Petri Nets, Reliability.

\section{Introduction.}

Stochastic comparison is a useful tool in the study of complex stochastic systems. Stoyan's book (1983) gives a broad survey on the subject. In this paper, the classical stochastic ordering is generalized to the comparison of processes through state functions. For example, in a Stochastic Petri Net (Murata (1989)) where tokens are moved randomly across the places, the total load of the system (sum of all marks) is a quantity of interest for the saturation conditions. Furthermore, a mark in a place can have a cost. The associated linear combination of marks, is a measure of the cost of the corresponding state system. Two processes obtained by changing the transition rates, can be stochastically compared through such state functions.

Let $\mathbf{X}=\left\{X_{t}, t \geq 0\right\}$ and $\mathbf{Y}=\left\{Y_{t}, t \geq 0\right\}$ be two Markov processes with values in $\mathrm{E}$ and $\mathrm{F}$ respectively and $\varphi, \psi$ two functions from $\mathrm{E}$ and $\mathrm{F}$ respectively into $\mathrm{G}$ (ordered by $\leq$ ). $\mathbf{X}$ and $\mathbf{Y}$ will be stochastically compared by means of $\varphi$ and $\psi$ using the stochastic ordering in G. We shall call this kind of comparison the $\varphi \psi$ comparison.

The comparison of functions of Markov processes with a stochastically monotone Markov process has been already studied by Massey (1987). Whitt (1986) compares general non-Markovian processes by assuming that they can be completed by additional information to become Markovian and always by mean of a stochastically monotone Markov process. We shall use a direct technique developed in particular in Liggett (1985) for interacting particle systems, the comparison by coupling. For 
countable state spaces the $\varphi \psi$ - comparability of processes is equivalent (Theorem 4) to the existence of a coupling Markov process with values in the product space $E \times F$ and which remains, at any instant, in the set

$$
K=\{(x, y) \in E \times F \quad \text { s.t. } \varphi(x) \leq \psi(y)\} .
$$

This technique is adapted only to the $\stackrel{\mathcal{D}}{\leq}$ stochastic comparison i.e. the comparison of probability measures through increasing functions (or our extension). But, combined with a cutting of transition rates (see section 4) it gives explicit comparison conditions. The construction of a particular coupling process is also given by the method. For simulations, it allows one to simulate only the coupling process which represents an important saving of computer's time. Moreover, for this coupling process staying in $K$, the comparison properties have a direct representation on the paths. Compared to the works of Massey and Whitt, this method avoids the use of monotone processes.

In section 2 the classical properties of the $\stackrel{\mathcal{D}}{\leq}$ comparison are extended to the $\varphi \psi-$ comparison. In section 3 the $\varphi \psi$ - comparison of Markov jump processes on countable spaces is introduced and the main characterizations of the $\varphi \psi$ - comparability are proved (Theorem 4 , Theorem 6 and Proposition 7).

The coupling technique for $\varphi \psi$ - comparison appears as a particular case of the following problem: the construction of a process with state space $E \times F$, with given marginal, and which stays in a fixed set $K$ for all times. Section 4 is devoted to this problem.

In the last section, examples are presented. At first, the classical comparison for processes with values in $I N$ are extented thanks to the $\varphi \psi$ - comparison for processes with state space $\mathbb{I}^{d}$. Then, an example in reliability theory is treated : degradable and repairable systems with redundant units are compared (directly or through some particular functions $\varphi$ and $\psi$ ). This example can also be treated by using recent results about stochastic comparison of interacting particle systems developed by Forbes et al. 1996. It is not surprising because most of these coupling methods for stochastic comparison, following Liggett, are based on the Vasershtein's coupling also called basic coupling (Lindvall p.177).

At last, we have to quote two papers on this subject (Zhang, S.Y. and Zhang, Y.H.) to appear in Acta Mathematica Sinica but not available at the present time.

\section{The $\varphi \psi$ - comparison of probability measures.}

Let $G$ be a Polish space endowed with its Borel $\sigma$-field $\mathcal{G}$ and a partial ordering denoted by $\leq$. Suppose that $\leq$ is closed i.e., the set

$$
K=\{(x, y) \in G \times G \text { s.t. } x \leq y\}
$$

is closed in the product topology on $G \times G$. A function $f$ from $G$ into $\mathbb{R}$ is increasing if $x \leq y$ implies $f(x) \leq f(y)$. A set $A$ is increasing if its indicator function, denoted 
by $\mathbb{1}_{A}$ is increasing.

For two probability measures $p$ and $p^{\prime}$ on $(G, \mathcal{G})$ the stochastic comparison denoted by $p \stackrel{\mathcal{D}}{\leq} p^{\prime}$ is defined by:

$$
\int_{G} f d p \leq \int_{G} f d p^{\prime} \text { for all } f \in \mathcal{M}_{i b}(G) .
$$

where $\mathcal{M}_{i b}(G)$ is the set of increasing bounded and measurable functions from $\mathrm{G}$ into $\mathbb{R}$. Recall the following result (see for example Lindvall (1994)).

If $p$ and $p^{\prime}$ are probability measures on $(G, \mathcal{G})$ such that $p \stackrel{\mathcal{D}}{\leq} p^{\prime}$ then, there exists a probability measure $\hat{p}$ on $(G \times G, \mathcal{G} \otimes \mathcal{G})$ with marginals $p$ and $p^{\prime}$ and such that $\hat{p}(K)=1$. The measure $\hat{p}$ is called a coupling (or increasing coupling) of $p$ and $p^{\prime}$. In the compact case, the proof can be obtained by the Riesz representation theorem and the Hahn-Banach theorem (see Lindvall (1992) or Liggett(1985)). But this theorem is also a special case of Theorem 11 in Strassen (1965), as pointed out by Marshall and Olkin (1979).

Let us consider now three Polish spaces with their Borel $\sigma$-fields $(E, \mathcal{E}),(F, \mathcal{F})$ and $(G, \mathcal{G})$. On $G$ a closed partial ordering $\leq$ is defined. Let $\varphi$ and $\psi$ be two continuous mappings from $E$ and $F$ into $G$. The $\varphi \psi$ - comparison between measures on $E$ and $F$ is defined as follows.

Definition 1 If $P$ and $P^{\prime}$ are two probability measures on $(E, \mathcal{E})$ and $(F, \mathcal{F})$ define

$$
P \leq_{\varphi \psi} P^{\prime} \quad \text { if and only if } \quad \varphi P \stackrel{\mathcal{D}}{\leq} \psi P^{\prime} .
$$

where $\varphi P$ is the image measure of $P$ by $\varphi$.

The notation $\leq_{\varphi \psi}$ may seem improprer. It is not an ordering between probability measures (defined on different spaces) but this notation recalls how this relation is defined. The following proposition characterizes the $\varphi \psi$ - comparison. It is an easy extension of the classical characterization of ordinary stochastic ordering.

Proposition 2 Suppose that $\psi(F) \subset \varphi(E)$. The two following assertions are equivalent:

i) $P \leq_{\varphi \psi} P^{\prime}$.

ii) There exists a coupling $\hat{P}$ on $(E \times F, \mathcal{E} \otimes \mathcal{F})$, with marginals $P$ and $P^{\prime}$ such that $\hat{P}(K)=1$ where

$$
K=\{(x, y) \in E \times F \text { s.t. } \varphi(x) \leq \psi(y)\} .
$$

Proof. It is clear that ii) implies $i$ ).

Let $G^{\prime}=\varphi(E)$ endowed with the order induced by $\leq$ and suppose now that $P \leq_{\varphi \psi} P^{\prime}$. The Strassen theorem (1965) (Theorem 11, p. 436) will be used with $\omega=K$ and $\varepsilon=0$. Let $U$ be an open set in $F$ and define $V=\pi_{E}[K \cap(E \times U)]$ 
where $\pi_{E}$ is the projection from $E \times F$ onto $E$. To establish the existence of $\hat{P}$ the inequality $P^{\prime}(U) \leq P(V)$ has to be proved.

For $x, x^{\prime}$ in $E$, we have

$$
x \in V \text { and } \varphi\left(x^{\prime}\right) \leq \varphi(x) \text { imply } x^{\prime} \in V .
$$

Then

$$
\mathbb{1}_{V}=\mathbb{1}_{\varphi(V)} \circ \varphi .
$$

and the function $\mathbb{1}_{\varphi(V)}$ is decreasing on $G^{\prime}$. Using $P \leq_{\varphi \psi} P^{\prime}$

$$
P(V)=\int_{E} \mathbb{1}_{\varphi(V)} \circ \varphi d P \geq \int_{F} \mathbb{1}_{\varphi(V)} \circ \psi d P^{\prime} .
$$

Now

$$
\mathbb{1}_{U} \leq \mathbb{1}_{\varphi(V)} \circ \psi .
$$

Indeed, take $u \in U$ and $z=\psi(u)$. Since $\psi(F) \subset \varphi(E), z=\varphi(x)$ with $(x, u)$ in $K \cap(E \times U), x$ in $V$ and $z$ in $\varphi(V)$. The inequality $P^{\prime}(U) \leq P(V)$ is proved.

As an immediate consequence of the existence of the coupling measure $\hat{P}$, the following result can be deduced.

Proposition 3 Under the hypothesis of Proposition (2), the following assertions are equivalent :

i) $P \leq_{\varphi \psi} P^{\prime}$

ii) For all $h \in \mathcal{M}_{b}(E), g \in \mathcal{M}_{b}(F)$ such that $h(x) \leq g(y)$ for $(x, y) \in K$

$$
\int_{E} h d P \leq \int_{F} g d P^{\prime}
$$

where $\mathcal{M}_{b}(E)$ denotes the set of bounded and measurable functions from $E$ into $\mathbb{R}$.

In the proof of Proposition (2) only indicator functions of increasing sets in $G^{\prime}$ were used. Taking $h=f \circ \varphi$ and $g=f \circ \psi$ in Proposition (3) with $f \in \mathcal{M}_{i b}\left(G^{\prime}\right)$ we have

$$
\int_{E} f \circ \psi d P \leq \int_{F} f \circ \varphi d P^{\prime},
$$

and then

$$
P \leq_{\varphi \psi} P^{\prime} .
$$

Thus, the $\leq_{\varphi \psi}$ stochastic comparison is entirely characterized by the increasing sets 
in $G^{\prime}$ (this is the point of view of Massey (1987)). Contrarily to the case of $\mathbb{R}$, it seems difficult, in any ordered set, to deduce directly the comparison of integrals of increasing functions from the comparison of probability measure of increasing sets. This result is one remarkable consequence of Strassen's Theorem.

For the classical $\stackrel{\mathcal{D}}{\leq}$ comparability, alternative formulations and a discrete time theorem for random sequences can be found in Lindvall (1994) (p.131 and Theorem 5.8 p.134). Using again the existence of $\hat{P}$, the same results still hold for the $\varphi \psi$ comparability. We shall not develop these easy extensions in order to focus on the $\varphi \psi$ - comparison of continuous time Markov processes.

\section{The $\varphi \psi$ - comparison of Markov processes.}

In what follows $E, F$ and $G$ are countable sets. The processes $\mathbf{X}=\left\{X_{t}, t \geq 0\right\}$ and $\mathbf{Y}=\left\{Y_{t}, t \geq 0\right\}$ are Markov jump processes with state spaces $E$ and $F$ respectively.

The transition semi-groups are denoted by $\mathbf{P}_{t}=\left\{P_{t}\left(x, x^{\prime}\right) / x \in E, x^{\prime} \in E\right\}$ and $\mathbf{P}^{\prime}{ }_{t}=\left\{P_{t}^{\prime}\left(y, y^{\prime}\right) / y \in F, y^{\prime} \in F\right\}$ and the initial distributions by $\mathcal{L}\left(X_{0}\right)$ and $\mathcal{L}\left(Y_{0}\right)$ respectively.

For all $x$ in $E, P_{t}(x, \cdot)$ is a probability measure on $E$ and $P_{t}$ an operator defined on $\mathcal{M}_{b}(E)$ by

$$
P_{t} f(x)=\sum_{x^{\prime} \in E} f\left(x^{\prime}\right) P_{t}\left(x, x^{\prime}\right) \quad \forall x \in E, \forall f \in \mathcal{M}_{b}(E) .
$$

If $\mathcal{L}\left(X_{0}\right)=\lambda$, the distribution of $X_{t}$ is the probability measure defined by

$$
\lambda \cdot P_{t}(A)=\sum_{x^{\prime} \in A} \sum_{x \in E} P_{t}\left(x, x^{\prime}\right) \lambda(x) \quad \forall A \subset E .
$$

The measure $\lambda \cdot P_{t}$ will be also denoted by $\mathcal{L}_{\lambda}\left(X_{t}\right)$. Here is the most natural extension of the classical stochastic comparison to transition semi-groups.

Definition 1 The $\varphi \psi$ - comparison of $\left\{\mathbf{P}_{t}\right\}$ and $\left\{\mathbf{P}_{t}^{\prime}\right\}$ is defined by

$$
\forall x \in E, \forall y \in F \quad \varphi(x) \leq \psi(y) \Longrightarrow P_{t}(x, \cdot) \leq_{\varphi \psi} P_{t}^{\prime}(y, \cdot) \quad, \quad \forall t \geq 0
$$

and will be denoted by

$$
\left\{\mathbf{P}_{t}\right\} \leq_{\varphi \psi}\left\{\mathbf{P}_{t}^{\prime}\right\} .
$$

For two Markov jump processes, the $\varphi \psi$ - comparison

$$
\mathbf{X} \leq_{\varphi \psi} \mathbf{Y}
$$

is defined by 


$$
\lambda \leq_{\varphi \psi} \mu \quad \text { and } \quad\left\{\mathbf{P}_{t}\right\} \leq_{\varphi \psi}\left\{\mathbf{P}_{t}^{\prime}\right\}
$$

Remark. In a Markovian setting, the main notion is the comparability of transition semi-groups. The condition on the initial distributions will sometimes be omitted for the comparison of processes.

The $\varphi \psi$ - comparability of initial distributions is preserved by $\varphi \psi$ - comparable transition semi-groups. Indeed, let $\lambda$ and $\mu$ be two probability measures on $E$ and $F$, a classical argument gives the following proposition.

Proposition 2 If $\left\{\mathbf{P}_{t}\right\} \leq_{\varphi \psi}\left\{\mathbf{P}_{t}^{\prime}\right\}$ then

$$
\lambda \leq_{\varphi \psi} \mu \quad \Longrightarrow \quad \lambda \cdot P_{t} \leq_{\varphi \psi} \mu \cdot P_{t}^{\prime} \quad, \quad \forall t \geq 0 .
$$

Let $\lambda \circ P_{t}$ denote the probability measure on $(E \times E, \mathcal{E} \otimes \mathcal{E})$ defined by

$$
\int_{A_{0}} \lambda(d x) P_{t}\left(x, A_{1}\right)
$$

for rectangles $A_{0} \times A_{1}$. More generally for $t_{1}<t_{2} \ldots<t_{n-1}\left(n \in N^{*}\right)$ the probability measure $\lambda \circ P_{t_{1}} \circ P_{t_{2}} \ldots \circ P_{t_{n-1}}$ can be defined by induction on $\left(E^{n}, \otimes^{n} \mathcal{E}\right)$. This is the distribution $\mathcal{L}\left(X_{0}, X_{t_{1}}, \ldots, X_{t_{n-1}}\right)$ of the vector $\left(X_{0}, X_{t_{1}}, \ldots, X_{t_{n-1}}\right)$. Let $\varphi^{\otimes^{n}}$ be the mapping from $E^{n}$ to $G^{n}$ defined by

$$
\left(x_{0}, \ldots, x_{n-1}\right) \longrightarrow\left(\varphi\left(x_{0}\right), \ldots, \varphi\left(x_{n-1}\right)\right) .
$$

On $G^{n}$ the partial ordering component by component and the associated stochastic comparison are considered. As can be expected, the definition (1) ensures the comparability of the finite dimensional distributions.

Proposition 3 If $\left\{\mathbf{P}_{t}\right\} \leq_{\varphi \psi}\left\{\mathbf{P}_{t}^{\prime}\right\}$ and $\lambda \leq_{\varphi \psi} \mu$ then

$$
\begin{aligned}
& \text { for all } t_{1}<t_{2} \ldots<t_{n-1} \quad\left(n \in \mathbb{N}^{*}\right) \\
& \lambda \circ P_{t_{1}} \circ P_{t_{2}} \ldots \circ P_{t_{n-1}} \leq_{\varphi_{\otimes^{n}}} \psi^{\otimes^{n}} \quad \mu \circ P_{t_{1}}^{\prime} \circ P_{t_{2}}^{\prime} \ldots \circ P_{t_{n-1}}^{\prime} \\
& \text { i.e., } \\
& \mathcal{L}\left(X_{0}, X_{t_{1}}, \ldots, X_{t_{n-1}}\right) \leq_{\varphi^{\otimes^{n}} \psi^{\otimes^{n}}} \quad \mathcal{L}\left(Y_{0}, Y_{t_{1}}, \ldots, Y_{t_{n-1}}\right)
\end{aligned}
$$

Proof. By induction with the same argument as in the Proposition 2.

A continuous time comparison theorem is developed in Kamae et al. (1977) for the $\stackrel{\mathcal{D}}{\leq}$ comparability of Markov processes with càdlàg paths in Polish spaces. Here is their result.

If for all $t \geq 0, P_{t}(x, \cdot) \stackrel{\mathcal{D}}{\leq} P_{t}^{\prime}(y, \cdot) \quad$ whenever $x \leq y$ and $\mathcal{L}\left(X_{0}\right) \stackrel{\mathcal{D}}{\leq} \mathcal{L}\left(Y_{0}\right)$ there 
exists Markov processes with càdlàg paths $\mathbf{X}=\left\{X_{t}, t \geq 0\right\}$ and $\mathbf{Y}=\left\{Y_{t}, t \geq 0\right\}$ defined on the same space, with initial distributions $\mathcal{L}\left(X_{0}\right)$ and $\mathcal{L}\left(Y_{0}\right)$ and with transition functions $\left\{\mathbf{P}_{t}\right\}$ and $\left\{\mathbf{P}_{t}^{\prime}\right\}$ such that:

$$
P\left(X_{t} \leq Y_{t}, \quad \forall t \geq 0\right)=1 .
$$

The proof is based on a discrete time comparison theorem. The two processes are first constructed for a countable dense subset of times. Then, their paths are extended to càdlàg paths. It is not difficult to see that the same argument can be used in our context. But in countable sets, a more precise result can be stated. Roughly speaking, the $\varphi \psi$ - comparability is equivalent to the existence of a coupling Markov process with values in $E \times F$ which stays for all $t$ in the set $K$. Conditions on the transition rates of the two processes that enable the construction of such a coupling process are given. Then, easily interpretable $\varphi \psi$ - comparability conditions are obtained. Recall that the transition rates are defined by:

$$
\begin{aligned}
q(x, y) & =\lim _{t \rightarrow 0^{+}} \frac{1}{t} P_{t}(x, y) \quad \text { for } x \neq y \\
q(x, x) & =-\sum_{y \neq x} q(x, y)
\end{aligned}
$$

The matrix $Q=(q(x, y)) / x \in E, y \in E)$ is the Q-matrix of the process (Anderson (1991)). We suppose that:

$$
\sup _{x \in E}|q(x, x)|<\infty
$$

and we denote $\|Q\|=\sup _{x \in E}|q(x, x)|$.

It is useful to see $Q$ as the infinitesimal generator of $\left\{\mathbf{P}_{t}\right\}$ that is for $f \in \mathcal{M}_{b}(E)$

$$
Q f(x)=\lim _{t \rightarrow 0^{+}} \frac{P_{t} f(x)-f(x)}{t},
$$

and also

$$
Q f(x)=\sum_{y \in E}[f(y)-f(x)] q(x, y) .
$$

The infinitesimal generator for $\mathbf{Y}$ will be denoted by $Q^{\prime}$. Here is the main result of this section.

Theorem 4 Suppose that $\psi(F) \subset \varphi(E)$.

The two following assertions are equivalent:

a) $\left\{\mathbf{P}_{t}\right\} \leq_{\varphi \psi}\left\{\mathbf{P}_{t}^{\prime}\right\}$.

b) For each probability measure $\lambda$ on $E$ and $\mu$ on $F$ such that $\lambda \leq_{\varphi \psi} \mu$, there exists a Markov jump process 


$$
\mathbf{Z}=\left\{Z_{t}=\left(\hat{X}_{t}, \hat{Y}_{t}\right), t \geq 0\right\}
$$

on $E \times F$ such that

i) $\hat{\mathbf{X}}$ (resp., $\hat{\mathbf{Y}}$ ) has $\mathbf{P}_{t}$ (resp., $\left.\mathbf{P}^{\prime}{ }_{t}\right)$ as transition semi-group and $\lambda($ resp., $\mu$ ) as initial distribution.

ii) $P\left(Z_{t} \in K, \quad \forall t \geq 0\right)=1$.

$\boldsymbol{Z}$ will be called a coupling process for $\boldsymbol{X}$ and $\boldsymbol{Y}$.

Proof. The only part to prove is the existence of the coupling process under the hypothesis of the theorem. The following classical representation for Markov jump processes (Çinlar (1975)) will be used for the construction of the coupling process. For $b \geq\|Q\|$, let $\left\{X_{n}^{b}\right\}$ be a Markov chain with transition kernel

$$
P^{b}=\mathbf{I}_{E}+\frac{1}{b} Q \quad\left(\mathbf{I}_{E} \quad \text { the identity matrix }\right)
$$

and let $\left\{N_{t}, t \geq 0\right\}$ be a Poisson process independent of the chain and with intensity $b$. Define $\left\{X_{t}^{b}, t \geq 0\right\}$ as the process obtained by indexing the chain $\left\{X_{n}^{b}\right\}$ by the Poisson process

$$
X_{t}^{b}=X_{N_{t}}^{b} .
$$

Then $\left\{X_{t}^{b}, t \geq 0\right\}$ is a Markov jump process with semi-group $\left\{\mathbf{P}_{t}\right\}$.

Lemma 5 If $\left\{\mathbf{P}_{t}\right\} \leq_{\varphi \psi}\left\{\mathbf{P}_{t}^{\prime}\right\} \quad$ and $\quad a \geq 2\left(\|Q\|+\left\|Q^{\prime}\right\|\right)$ then

$$
\varphi(x) \leq \psi(y) \quad \Longrightarrow \quad P^{a}(x, \cdot) \leq_{\varphi \psi} P^{\prime a}(y, \cdot)
$$

Proof. As already noted, the increasing sets characterize the $\leq_{\varphi \psi}$ stochastic ordering. Hence, it suffices to prove that for any increasing set $\Gamma$ in $G$

$$
\varphi P^{a}(x, \Gamma) \leq \psi P^{\prime a}(y, \Gamma)
$$

for $\varphi(x) \leq \psi(y)$ and $a \geq 2\left(\|Q\|+\left\|Q^{\prime}\right\|\right)$.

The following equation is a direct consequence of the definition of $P^{a}$.

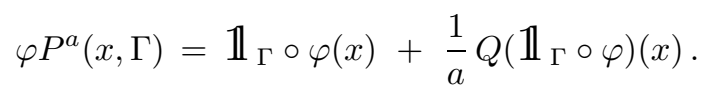

1) Suppose first that $\varphi(x) \in \Gamma$ or $\psi(y) \notin \Gamma$.

The set $\Gamma$ is an increasing set and $\varphi(x) \leq \psi(y)$, so in this case

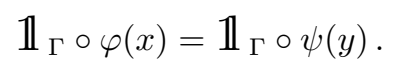

As $\left\{\mathbf{P}_{t}\right\} \leq_{\varphi \psi}\left\{\mathbf{P}_{t}^{\prime}\right\}$ one has

$$
P_{t}\left(\mathbb{1}_{\Gamma} \circ \varphi\right)(x) \leq P_{t}^{\prime}\left(\mathbb{1}_{\Gamma} \circ \psi\right)(y)
$$


and then

$$
P_{t}\left(\mathbb{1}_{\Gamma} \circ \varphi\right)(x)-\mathbb{1}_{\Gamma} \circ \varphi(x) \leq P_{t}^{\prime}\left(\mathbb{1}_{\Gamma} \circ \psi\right)(y)-\mathbb{1}_{\Gamma} \circ \psi(y) .
$$

Dividing by $\mathrm{t}$ and letting $\mathrm{t}$ tend to $0^{+}$we deduce that

$$
Q\left(\mathbb{1}_{\Gamma} \circ \varphi\right)(x) \leq Q^{\prime}\left(\mathbb{1}_{\Gamma} \circ \psi(y)\right),
$$

which proves (3) by (4).

By (4)

2) The only remaining case is : $\mathbb{1}_{\Gamma} \circ \varphi(x)=0$ and $\mathbb{1}_{\Gamma} \circ \psi(y)=1$.

$$
\begin{aligned}
\varphi P^{a}(x, \Gamma)-\psi{P^{\prime}}^{a}(y, \Gamma) & \leq \frac{1}{a}\left|Q\left(\mathbb{1}_{\Gamma} \circ \varphi\right)(x)-Q^{\prime}\left(\mathbb{1}_{\Gamma} \circ \psi\right)(y)\right|-1 . \\
& \leq \frac{2}{a}\left(\|Q\|+\left\|Q^{\prime}\right\|\right)-1 . \\
& \leq 0 \text { for } a \geq 2\left(\|Q\|+\left\|Q^{\prime}\right\|\right) .
\end{aligned}
$$

Therefore, it suffices to deal with the $\varphi \psi$ stochastic comparison of transition kernels for $a$ sufficiently large. In the countable case it is easy to couple such kernels to stay in $K$ after reaching $K$. A Markov jump process which jumps according to this coupled transition kernel at the instants of an independent Poisson process of intensity $a$ can then be defined. The components of this process move as $\mathbf{X}$ and $\mathbf{Y}$ independently of $a$ and stay at all times in $K$ after reaching $K$.

More precisely, for $a \geq 2\left(\|Q\|+\left\|Q^{\prime}\right\|\right)$ define $R^{a}[(x, y),(\cdot, \cdot)]$ as:

* the coupling measure of $P^{a}(x, \cdot)$ and $P^{\prime a}(y, \cdot)$ when $(x, y) \in K$ (by Theorem 2)

* the product measure $P^{a}(x, \cdot) \otimes P^{\prime a}(y, \cdot)$ if $\quad(x, y) \notin K$.

Then $R^{a}$ is the transition kernel of a Markov Chain on $E \times F$.

If $\tilde{Q}_{a}=a\left(R^{a}-\mathbf{I}_{E \times F}\right)$ and $\mathbf{R}_{t}^{a}=\exp \left(t \tilde{Q}_{a}\right)$ then $\left\{\mathbf{R}_{t}^{a}, t \geq 0\right\}$ is a Markov semi-group on $E \times F$. The construction of $R^{a}$ implies easily that:

1) if $f \in \mathcal{M}_{b}(E \times F)$ with $f(x, y)=h(x) \quad \forall x \in E, \forall y \in F$, then

$$
\tilde{Q}_{a} f(x, y)=Q f(x) \quad \forall x \in E, \forall y \in F .
$$

(and symmetrically for $f(x, y)=k(y) \quad \forall x \in E, \forall y \in F$ ).

$$
\tilde{Q}_{a}\left[\mathbb{1}_{K^{c}}\right](x, y)=0 \quad \forall(x, y) \in K .
$$

Let $\nu$ be a coupling measure for $\lambda$ and $\mu$ and $\mathbf{Z}^{a}=\left\{\left(\hat{X}_{t}^{a}, \hat{Y}_{t}^{a}\right), t \geq 0\right\}$ a Markov jump process with values in $E \times F$, transition semi-group $\left\{\mathbf{R}_{t}^{a}\right\}$ and initial distribution $\nu$. Due to 1) $\left\{\hat{X}_{t}^{a}, t \geq 0\right\}$ and $\left\{\hat{Y}_{t}^{a}, t \geq 0\right\}$ are Markov jump process with transition semi-group $\left\{\mathbf{P}_{t}\right\}$ and $\left\{\mathbf{P}_{t}^{\prime}\right\}$ and initial distributions $\lambda$ and $\mu$ (Liggett 
(1985) Theorem 1.3 p. 126). By 2) the process starting in $K$, stays in $K$ for all $t$. As already noted, the parameter $a$ is only used for the representation of the coupling process as a Markov chain timed by a Poisson process. In other words, for different values of $a$ (larger than $2\left(\|Q\|+\left\|Q^{\prime}\right\|\right)$ ) the processes $\mathbf{Z}^{a}$ have actually the same behavior and define the same coupling process.

A simple characterization of the $\varphi \psi$ - comparability of semi-groups through their generators is now given. This characterization is analogous to that of Massey (1987) , here in a more general context.

TheOREM $6 \quad$ Suppose that $\psi(F) \subset \varphi(E)$.

The two following assertions are equivalent:

i) $\left\{\mathbf{P}_{t}\right\} \leq_{\varphi \psi}\left\{\mathbf{P}_{t}^{\prime}\right\}$.

ii) for each increasing set $\Gamma$ in $G$ and for each $(x, y)$ in $K$ s.t. $\varphi(x) \in \Gamma$ or $\psi(y) \notin \Gamma$ we have

$$
Q\left[\mathbb{1}_{\Gamma} \circ \varphi\right](x) \leq Q^{\prime}\left[\mathbb{1}_{\Gamma} \circ \psi\right](y) .
$$

\section{Proof.}

i) $\Rightarrow$ ii) It is the first part of the proof of Lemma 5 .

ii) $\Rightarrow$ i) If ii) is true, we have, as in Lemma 5

$$
\varphi(x) \leq \psi(y) \quad \Longrightarrow \quad P^{a}(x, \cdot) \leq_{\varphi \psi} P^{\prime a}(y, \cdot)
$$

for $a \geq 2\left(\|Q\|+\left\|Q^{\prime}\right\|\right)$. Indeed, the second part of the proof of this Lemma, depends only on the condition $a \geq 2\left(\|Q\|+\left\|Q^{\prime}\right\|\right)$. Then, the construction of the coupling process $\mathbf{Z}$ of Theorem (4) is possible for all $\lambda$ and $\mu, \varphi \psi$ comparable and we have

$$
\left\{\mathbf{P}_{t}\right\} \leq_{\varphi \psi}\left\{\mathbf{P}_{t}^{\prime}\right\}
$$

As particular case, let $\mathbf{X}=\left\{X_{t}, t \geq 0\right\}$ be a continuous-time non Markovian jump process with state space G. Suppose that, with the additional information of $\left\{X_{t}^{\prime}\right\}$ the process $\tilde{\mathbf{X}}=\left\{\left(X_{t}, X_{t}^{\prime}\right), t \geq 0\right\}$ becomes a Markov jump process with state space $G \times G^{\prime}$. Let $\mathbf{Y}=\left\{Y_{t}, t \geq 0\right\}$ be another Markov jump process with state space $G$. If $\pi$ is the first projection from $G \times G^{\prime}$ onto $G$ and $i$ the identity mapping of $G$, the $\leq_{\pi i}$ stochastic comparison between $\tilde{\mathbf{X}}$ and $\mathbf{Y}$ is exactly the comparison introduced by Whitt (1986) to compare the non-Markovian process $\mathbf{X}$ with the Markovian one $\mathbf{Y}$ in the particular case of $\stackrel{\mathcal{D}}{\leq}$ stochastic comparison in $G$. In the setting of Whitt, the process $\mathbf{Y}$ must be supposed $\stackrel{\mathcal{D}}{\leq}$ - monotone, restriction that we do not need. On the other hand, Whitt's comparison can be used with more general stochastic orderings in $G$. Yet, if $\mathbf{Y}$ is $\stackrel{\mathcal{D}}{\leq}$ - monotone, the conditions $i i)$ of Theorem (6) are equivalent to Whitt's conditions (1986 p. 614).

The following translation in terms of transition rates will be of fundamental importance in the sequel. Indeed, our aim is to obtain explicit conditions for stochastic comparison and the possibility to construct the coupling process. 
A Markov jump process $\mathbf{Z}$ with infinitesimal generator $\tilde{Q}$ on $E \times F$ stays always in $K$ after reaching $K$ if and only if the rates between $K$ and $K^{c}$ are null i.e.

$$
\tilde{q}\left[(x, y),\left(x^{\prime}, y^{\prime}\right)\right]=0 \quad \forall(x, y) \in K, \forall\left(x^{\prime}, y^{\prime}\right) \in K^{c} .
$$

On the other hand, the components $\hat{\mathbf{X}}$ and $\hat{\mathbf{Y}}$ of $\mathbf{Z}$ are Markov jump processes with infinitesimal generators $Q$ and $Q^{\prime}$ if and only if

$$
\begin{aligned}
& \sum_{y^{\prime} \in F} \tilde{q}\left[(x, y),\left(x^{\prime}, y^{\prime}\right)\right]=q\left(x, x^{\prime}\right) \quad \forall x, x^{\prime} \in E, \forall y \in F . \\
& \sum_{x^{\prime} \in E} \tilde{q}\left[(x, y),\left(x^{\prime}, y^{\prime}\right)\right]=q^{\prime}\left(y, y^{\prime}\right) \quad \forall y, y^{\prime} \in F, \forall x \in E .
\end{aligned}
$$

(see Anderson (1991) p. 256-257). So Theorem 4 can be expressed on $Q$ and $Q^{\prime}$.

Proposition 7 The two following assertions are equivalent:

i) $\left\{\mathbf{P}_{t}\right\} \leq_{\varphi \psi}\left\{\mathbf{P}_{t}^{\prime}\right\}$.

ii) There exists an infinitesimal generator $\tilde{Q}$ on $E \times F$ such that (6), (7) and (8) hold.

If this is the case, we shall write

$$
Q \leq_{\varphi \psi} Q^{\prime} .
$$

Intuitively $Q$ and $Q^{\prime}$ are $\varphi \psi$ - comparable, if the transition rates can be partitioned in such a way that (6), (7) and (8) hold.

\section{The general coupling technique.}

By Proposition (7) a way to construct a coupling generator, is proposed. It seems interesting to try a sketch of general theory of coupling.

Let $\mathbf{X}=\left\{X_{t}, t \geq 0\right\}$ and $\mathbf{Y}=\left\{Y_{t}, t \geq 0\right\}$ be two Markov jump processes with state spaces $E$ and $F$ and generators $Q$ and $Q^{\prime}$, if $K$ is a subset of $E \times F$, what are the conditions on $Q$ and $Q^{\prime}$ to be able to construct $\tilde{Q}$ on $E \times F$ such that (6), (7) and (8) are satisfied ?

Then, a Markov jump process say $\mathbf{Z}=\left\{Z_{t}=\left(\hat{X}_{t}, \hat{Y}_{t}\right), t \geq 0\right\}$ with generator $\tilde{Q}$ and initial distribution concentraded on $K$ will stay in $K$ for all $t$; moreover $\left\{\hat{X}_{t}\right\}$ and $\left\{\hat{Y}_{t}\right\}$ are also Markov jump processes with generators $Q$ and $Q^{\prime}$. Such a process will be called a coupling process associated to $K$.

Outside $K$, the two coordinates move independently and with the marginal rates. For some points in $K$, the jumps of the two coordinates cannot be independent, if we want then to stay in $K$. The idea of the coupling is the following : if we are going out of $K$ by a jump of the first coordinate, we must balance it by a jump of the second coordinate, in such a way that the final point is in $K$. In addition, for 
all points, the marginal rates must be respected (conditions $(7),(8)$ ).

The numerical values of the rates, but also the geometry of $K$ are important in this construction. It seems too ambitious to give a completely general construction (when it is possible) of a coupling process. At first, some additional conditions are assumed. They allow us to describe the cutting technique of the transitions rates. Then, examples where the preceding hypotheses fail are treated. They show how this technique can be easily adapted to each particular case, after a look at the local situation of each point of $K$ for the two processes.

Define for all $(x, y)$ in $K$

$$
\mathcal{O}_{1}(x, y)=\left\{z \in E \text { s.t. } q(x, z) \neq 0 \text { and }(z, y) \in K^{c}\right\}
$$

i.e. the possible jumps out of $K$ by the first coordinate from the point $(x, y)$ $\left(\mathcal{O}_{2}(x, y)\right.$ is defined symmetrically).

Now, for $z \in \mathcal{O}_{1}(x, y)$ define

$$
\mathcal{J}_{2}(x, y ; z)=\left\{v \in F \text { s.t. } q^{\prime}(y, v) \neq 0 \text { and }(z, v) \in K\right\}
$$

i.e. the compensating jumps of the second coordinate, which allow to stay in $K$ when the first coordinate jumps from $x$ to $z\left(\mathcal{J}_{1}(x, y ; w)\right.$ for $w \in \mathcal{O}_{2}(x, y)$ is defined symmetrically).

Then, the jumps $(x, y) \rightarrow(z, v)$ with $v \in \mathcal{J}_{2}(x, y ; z)$ replaces if possible, the forbidden jump $(x, y) \rightarrow(z, y)$.

The following assumptions are assumed.

$\left(\mathbf{H}_{\mathbf{1}}\right) \quad$ The jumps which allow to compensate a departure from $K$ by the first coordinate (resp., the second), are the same for all $z$ in $\mathcal{O}_{1}(x, y)$ (resp., for all $w$ in $\left.\mathcal{O}_{2}(x, y)\right)$.

Then, it can be written

$$
\begin{array}{ll}
\mathcal{J}_{1}(x, y) \stackrel{\text { def }}{=} \mathcal{J}_{1}(x, y ; w) & \forall w \in \mathcal{O}_{2}(x, y) \\
\mathcal{J}_{2}(x, y) \stackrel{\text { def }}{=} \mathcal{J}_{2}(x, y ; z) & \forall z \in \mathcal{O}_{1}(x, y)
\end{array}
$$

$\left(\mathbf{H}_{\mathbf{2}}\right) \quad$ The jumps which can compensate a departure from $K$ by $z$ in $E$, are not those, which alone, will provoke an exit from $K$ i.e.

$$
\begin{aligned}
& \mathcal{J}_{2}(x, y) \cap \mathcal{O}_{2}(x, y)=\emptyset \\
& \mathcal{J}_{1}(x, y) \cap \mathcal{O}_{1}(x, y)=\emptyset
\end{aligned}
$$

Moreover, from all states $x \in E$ (resp., $y \in F$ ) the set of jumps with non null transition rates, are supposed to be finite. It is the case of interest for our applications. However, the construction is valid in the countable case. 
ThEOREM 1 A coupling process associated to $K$ can be constructed if and only if for all $(x, y)$ in $K$ :

$$
\sum_{u \in \mathcal{O}_{1}(x, y)} q(x, u) \leq \sum_{v \in \mathcal{J}_{2}(x, y)} q^{\prime}(y, v)
$$

and symmetrically

$$
\sum_{u \in \mathcal{J}_{1}(x, y)} q(x, u) \geq \sum_{v \in \mathcal{O}_{2}(x, y)} q^{\prime}(y, v) .
$$

(By convention, a sum on an empty set is null).

Proof. These conditions are necessary. Indeed, if $\tilde{Q}$ satisfying $(6),(7),(8)$ does exist, we can write successively:

$$
\begin{aligned}
\sum_{z \in \mathcal{O}_{1}(x, y)} q(x, z) & =\sum_{z \in \mathcal{O}_{1}(x, y)}\left[\sum_{v \in F} \tilde{q}[(x, y),(z, v)]\right] \text { by }(7) \\
& =\sum_{z \in \mathcal{O}_{1}(x, y)}\left[\sum_{v \in \mathcal{J}_{2}(x, y)} \tilde{q}[(x, y),(z, v)]\right] \text { by }(6) \text { and }\left(\mathbf{H}_{\mathbf{1}}\right) \\
& =\sum_{v \in \mathcal{J}_{2}(x, y)}\left[\sum_{z \in \mathcal{O}_{1}(x, y)} \tilde{q}[(x, y),(z, v)]\right] \\
& \leq \sum_{v \in \mathcal{J}_{2}(x, y)} q^{\prime}(y, v) \text { by }(8) .
\end{aligned}
$$

The conditions of Theorem (1) are sufficient. The coupling process associated to $K$, will be constructed by its generator $\tilde{A}$ on $\mathcal{E}_{b}(E \times F)$. An elementary technical lemma is the key of the cutting technique.

Lemma 2 Let $d$ and $d^{\prime}$ be two integers and $\alpha_{1}, \ldots, \alpha_{d}$ and $\beta_{1}, \ldots, \beta_{d^{\prime}}$ two families of non negative real numbers such that

$$
\alpha_{1}+\cdots+\alpha_{d} \leq \beta_{1}+\cdots+\beta_{d^{\prime}} .
$$

Then, the non negative real numbers $u_{m l}$ defined by:

$$
u_{m l}=\frac{\alpha_{l} \beta_{m}}{\beta_{1}+\cdots+\beta_{d^{\prime}}}
$$

satisfy

$$
\begin{aligned}
& \sum_{m=1}^{d^{\prime}} u_{m l}=\alpha_{l} \quad \forall l=1, \ldots, d \\
& \sum_{l=1}^{d} u_{m l} \leq \beta_{m} \quad \forall m=1, \ldots, d^{\prime} .
\end{aligned}
$$


Consider a function $f$ in $\mathcal{E}_{b}(E \times F)$. We define $\tilde{A} f(x, y)$ for all $(x, y)$ in $E \times F$ as follows.

If $(x, y) \notin K$. Each coordinate moves independently with the marginal rates.

If $(x, y) \in K$ with $\mathcal{O}_{1}(x, y)=\emptyset$ and $\mathcal{O}_{2}(x, y)=\emptyset$ (i.e. no departure from $\left.K\right)$. The coupling process is defined as in the preceding case.

If $(x, y) \in K$ with $\mathcal{O}_{1}(x, y) \cup \mathcal{O}_{2}(x, y) \neq \emptyset$. From $x$, three disjoint sets of jumps have to be considered (by $\left.\left(\mathbf{H}_{2}\right)\right): \mathcal{O}_{1}(x, y), \mathcal{J}_{1}(x, y)$ and the the set of the others. The same is true for $y$. Then, a corresponding partition of the generator in two pieces is allowed. Only the first one, say $[\tilde{A} f(x, y)]_{1}$, is now given. Suppose that $\mathcal{O}_{1}(x, y)=\left\{x_{1}, \ldots, x_{d}\right\}$ and $\mathcal{J}_{2}(x, y)=\left\{y_{1}, \ldots, y_{d^{\prime}}\right\}$

By hypothesis, we have:

$$
\sum_{l=1}^{d} q\left(x, x_{l}\right) \leq \sum_{m=1}^{d^{\prime}} q^{\prime}\left(y, y_{m}\right)
$$

By lemma (2), we can find $u_{m l}, m=1, \ldots, d^{\prime}, l=1, \ldots, d$ such that:

$$
\begin{aligned}
& \sum_{m=1}^{d^{\prime}} u_{m l}=q\left(x, x_{l}\right) \quad \forall l=1, \ldots d . \\
& \sum_{l=1}^{d} u_{m l} \leq q^{\prime}\left(y, y_{m}\right) \quad \forall m=1, \ldots, d^{\prime} .
\end{aligned}
$$

Then $[\tilde{A} f(x, y)]_{1}$ is defined by

$$
\begin{aligned}
{[\tilde{A} f(x, y)]_{1} } & =\sum_{m=1}^{d^{\prime}} \sum_{l=1}^{d} u_{m l}\left[f\left(x_{l}, y_{m}\right)-f(x, y)\right] \\
& +\sum_{m=1}^{d^{\prime}}\left[q^{\prime}\left(y, y_{m}\right)-\sum_{l=1}^{d} u_{m l}\right]\left[f\left(x, y_{m}\right)-f(x, y)\right] \\
& +\sum_{u \notin \mathcal{J}_{1}(x, y) \cup \mathcal{O}_{1}(x, y)} q(x, u)[f(u, y)-f(x, y)] .
\end{aligned}
$$

By (10) all the rates are positive, no jump can take the process out of $K$ and from (9) and the definition of $[\tilde{A} f(x, y)]_{1}$ the marginal rates are preserved.

Then (6), (7) and (8) are satisfied. 
We shall now study some situations where $\left(\mathbf{H}_{\mathbf{1}}\right)$ and $\left(\mathbf{H}_{\mathbf{2}}\right)$ fail.

First case.(See Figure 1) From $x$, the jumps $x \rightarrow x_{1}, x \rightarrow x_{2}$ and $x \rightarrow x_{3}$ are possible and from $y$ the jumps $y \rightarrow y_{1}$ and $y \rightarrow y_{2}$ are possible. The vectors represent the only possible jumps from $(x, y)$ to stay in $K$.

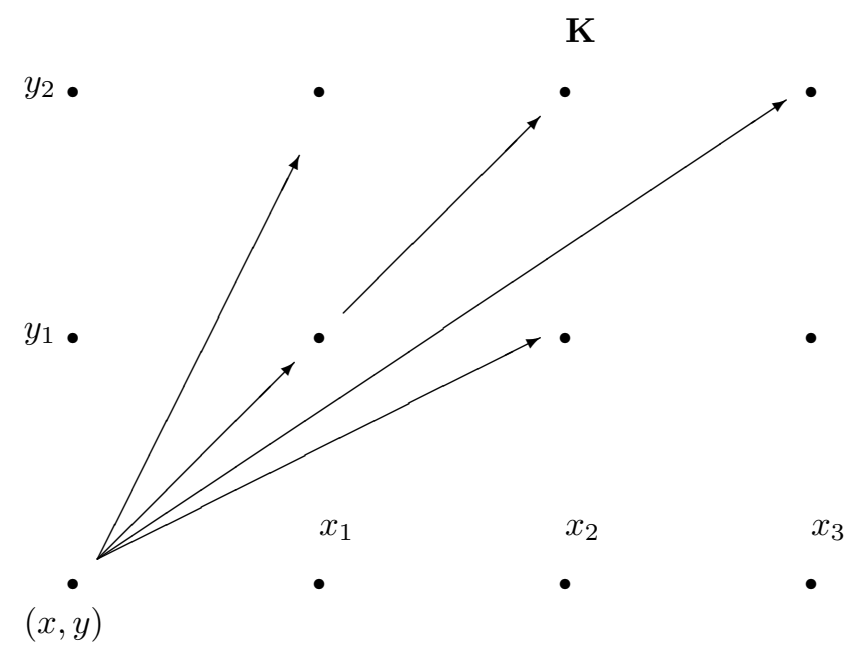

Figure 1

Here, $\mathcal{O}_{1}(x, y)=\left\{x_{1}, x_{2}, x_{3}\right\} ; \mathcal{J}_{2}\left(x, y ; x_{1}\right)=\mathcal{J}_{2}\left(x, y ; x_{2}\right)=\left\{y_{1}, y_{2}\right\}$

and $\mathcal{J}_{2}\left(x, y ; x_{3}\right)=\left\{y_{2}\right\}$. Hypothesis $\left(\mathbf{H}_{2}\right)$ is true. To stay in $K$, the jump $(x, y) \rightarrow\left(x_{3}, y\right)$ must be replaced by the jump $\left.(x, y) \rightarrow\left(x_{3}, y_{2}\right)\right)$. It is possible if and only if:

$$
q\left(x, x_{3}\right) \leq q^{\prime}\left(y, y_{2}\right)
$$

The jump $y \rightarrow y_{2}$ can still be used to compensate a departure from $K$ with a residual rate: $q^{\prime}\left(y, y_{2}\right)-q\left(x, x_{3}\right)$. Then, the second condition to compensate the jumps $x \rightarrow x_{1}$ and $x \rightarrow x_{2}$ is:

$$
\begin{aligned}
q\left(x, x_{1}\right)+q\left(x, x_{2}\right) & \leq q^{\prime}\left(y, y_{1}\right)+\left[q^{\prime}\left(y, y_{2}\right)-q\left(x, x_{3}\right)\right] \\
& \text { or } \\
q\left(x, x_{1}\right)+q\left(x, x_{2}\right)+q\left(x, x_{3}\right) & \leq q^{\prime}\left(y, y_{1}\right)+q^{\prime}\left(y, y_{2}\right) .
\end{aligned}
$$


In this situation, the construction of the coupling process is possible if and only if the conditions (11) and (11) are satisfied.

Second case. (See Figure 2) The same conventions are made.

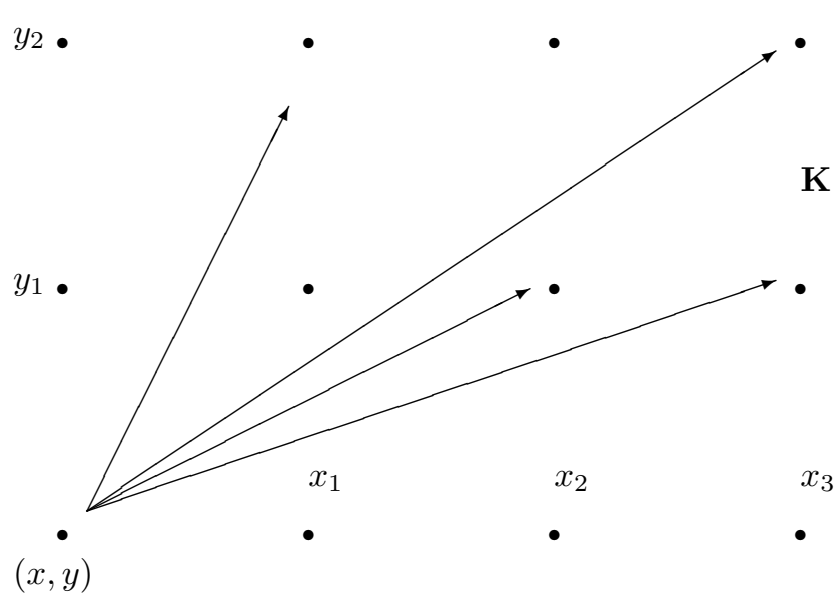

Figure 2

Here, $\mathcal{O}_{1}(x, y)=\left\{x_{1}, x_{2}, x_{3}\right\} ; \mathcal{J}_{2}\left(x, y ; x_{1}\right)=\left\{y_{2}\right\} ; \mathcal{J}_{2}\left(x, y ; x_{2}\right)=\left\{y_{1}\right\}$ and $\mathcal{J}_{2}\left(x, y ; x_{3}\right)=\left\{y_{1}, y_{2}\right\}$.

Hypothesis $\left(\mathbf{H}_{2}\right)$ is still true. For the jumps: $x \rightarrow x_{1}$ and $x \rightarrow x_{2}$, the first two conditions are:

$$
\begin{aligned}
& q\left(x, x_{1}\right) \leq q^{\prime}\left(y, y_{2}\right) \\
& q\left(x, x_{2}\right) \leq q^{\prime}\left(y, y_{1}\right)
\end{aligned}
$$

For $x \rightarrow x_{3}$, the two jumps $y \rightarrow y_{1}$ and $y \rightarrow y_{2}$ can be used again, with residual rates $q^{\prime}\left(y, y_{1}\right)-q\left(x, x_{2}\right)$ and $q^{\prime}\left(y, y_{2}\right)-q\left(x, x_{1}\right)$. Then, the third condition is :

$$
q\left(x, x_{3}\right) \leq q^{\prime}\left(y, y_{1}\right)-q\left(x, x_{2}\right)+q^{\prime}\left(y, y_{2}\right)-q\left(x, x_{1}\right)
$$

or

$$
q\left(x, x_{1}\right)+q\left(x, x_{2}\right)+q\left(x, x_{3}\right) \leq q^{\prime}\left(y, y_{1}\right)+q^{\prime}\left(y, y_{2}\right)
$$


The conditions (13) , (14) and (15) are necessary and sufficient to be able to construct the coupling process in $(x, y)$.

If $\left(\mathbf{H}_{\mathbf{2}}\right)$ is not true, the second term in $[\tilde{A} f(x, y)]_{1}$ cannot be used and the conditions become immediately very restrictive. Consider the following situation:

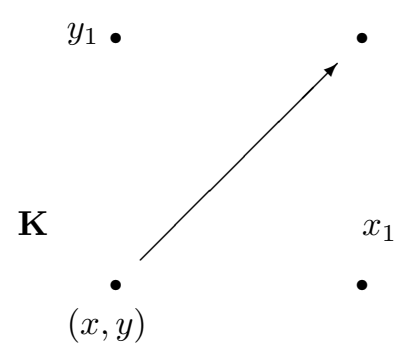

Figure 3

The jumps $(x, y) \rightarrow\left(x_{1}, y\right)$ and $(x, y) \rightarrow\left(x, y_{1}\right)$ can only be replaced by: $(x, y) \rightarrow$ $\left(x_{1}, y_{1}\right)$ and this is possible if and only if:

$$
q\left(x, x_{1}\right)=q^{\prime}\left(y, y_{1}\right) .
$$

In conclusion, this technique gives a practical way to obtain the necessary and sufficient conditions for constructing the coupling process for a large class of sets $K$. And then, a way to construct the coupling process itself. For the $\leq_{\varphi \psi}$ comparison, the set $K$ will be:

$$
K=\{(x, y) \in E \times F \text { s.t. } \varphi(x) \leq \psi(y)\} .
$$

Examples of pratical interest are developped in the next section.

\section{Examples.}

This section is devoted to examples. At first, the well-known stochastic comparison conditions for Markov jump process with state space $I N$ are recalled. A coupling point of view of these results and extensions for $\leq_{\varphi \psi}$ comparison for Stochastic Petri Nets are given. The second example is a complete analysis of one degradable and reparable system with multiple component types and redundant units, already introduced by Cocozza et Roussignol (1995) . Our approach is used to obtain necessary and sufficient conditions for the ordinary $\stackrel{\mathcal{D}}{\leq}$ stochastic comparison. Then, 
some natural functions $\varphi$ and $\psi$ are introduced and the associated $\leq_{\varphi \psi}$ comparison is examined.

Example 1. Consider a Markov jump process with state space $\mathbb{I N}$. Suppose that from each $n \in \mathbb{N}$, the only possible jumps are:

$$
n-p, \cdots, n-1, n+1, \cdots, n+r .
$$

with $r$ and $p$ fixed in $\mathbb{N}^{*}$. Such a process will be called for commodity in the sequel a $r$-p birth and death process. IN is endowed with the ordinary ordering. The corresponding stochastic comparison for processes will be denoted by $\stackrel{\mathcal{D}}{\leq}$. Let $\mathbf{X}=\left\{X_{t}, t \geq 0\right\}$ and $\mathbf{Y}=\left\{Y_{t}, t \geq 0\right\}$ be two such $r$ - $p$ birth and death processes.

\section{Proposition $1 \quad \mathrm{X} \stackrel{\mathcal{D}}{\leq} \mathrm{Y}$}

if and only if $\quad \forall(n, m) \in \mathbb{N} \times \mathbb{N}$ s.t. $n \leq m$

$$
\begin{array}{ll}
\forall v>m & \sum_{u \text { s.t. } u \geq v} q(n, u) \leq \sum_{u \text { s.t. } u \geq v} q^{\prime}(m, u) \\
\forall v<m \quad \sum_{u \text { s.t. } u \leq v} q(n, u) \geq \sum_{u \text { s.t. } u \leq v} q^{\prime}(m, u) .
\end{array}
$$

This is a particular case of Theorem 4.6 .8 p.67 in Stoyan (1983). A coupling argument in the line of this paper can be given. Begin with the maximal jump $n \rightarrow n+r$ for $\mathbf{X}$. $\mathbf{Y}$ must jump to $u \geq n+r$ and the first condition is:

$$
q(n, n+r) \leq \sum_{u \text { s.t. } u \geq n+r} q^{\prime}(m, u) .
$$

This is (16) for $v=n+r$.

If $\mathbf{X}$ jumps to $n+r-1, \mathbf{Y}$ can jump to $u \geq n+r$ with the residual rate

$$
\sum_{u s . t . u \geq n+r} q^{\prime}(m, u)-q(n, n+r)
$$

or to $n+r-1$ with the rate $q^{\prime}(m, n+r-1)$. Then, the second condition is:

$$
q(n, n+r-1) \leq\left[\sum_{u \text { s.t. } u \geq n+r} q^{\prime}(m, u)-q(n, n+r)\right]+q^{\prime}(n, n+r-1)
$$

i.e.,

$$
q(n, n+r-1)+q(n, n+r) \leq \sum_{u \text { s.t. } u \geq n+r-1} q^{\prime}(m, u) .
$$


This is (16) for $v=n+r-1$. And so on.

Consider now a Markov jump process $\mathbf{X}=\left\{X_{t}, t \geq 0\right\}$ with values in a countable set $E$ and $\varphi$ a function from $E$ to $\mathbb{I N}$ such that the only possible jumps of $\varphi(\mathbf{X})$ is from $n$ to

$$
n-p, \cdots, n-1, n+1, \cdots, n+r .
$$

Then, $\varphi(\mathbf{X})$ behaves as a $r$ - $p$ birth and death process. For example if $\mathbf{X}$ is a Markovian Petri Net $E=\mathbb{N}^{d}$, and $\mathbf{X}$ has only a finitely many possible jumps from each state. The function $\varphi$ can be for instance the sum of all coordinates. The same assumptions are made on $\mathbf{Y}=\left\{Y_{t}, t \geq 0\right\}$ with state space $F$ and $\psi$ from $F$ to $\mathbb{I N}$.

¿From $X_{t}=x, x \in E$ and $\varphi\left(X_{t}\right)=n, n \in \mathbb{N}$, the total transition rate to $m \in \mathbb{N}$ is

$$
\sum_{\varphi(z)=m} q(x, z) .
$$

Then define:

$$
\lambda(x, u)=\sum_{\varphi(z)=\varphi(x)+u} q(x, z) \quad x \in E, u \in \mathbb{N} .
$$

If $(x, y) \in K$ i.e., $\varphi(x) \leq \psi(y)$, our coupling argument shows that the $\varphi \psi$ - comparison conditions reduce to the ordinary stochastic comparison of $r$ - $p$ birth and death processes, with rates $\lambda(x, u)$ and $\lambda^{\prime}(y, v)$.

\section{Proposition $2 \quad \mathrm{X} \leq_{\varphi \psi} \mathbf{Y}$}

if and only if

$\forall n, m \in \mathbb{N}$ s.t. $n \leq m$ and $\forall x \in E, \forall y \in F$ s.t $n=\varphi(x)$ and $\psi(y)=m$

$$
\begin{array}{ll}
\forall v>m & \sum_{u \text { s.t. } u \geq v} \lambda(x, u) \leq \sum_{u \text { s.t. } u \geq v} \lambda^{\prime}(y, u) \\
\forall v<m \quad \sum_{u \text { s.t. } u \leq v} \lambda(x, u) \geq \sum_{u \text { s.t. } u \leq v} \lambda^{\prime}(y, u) .
\end{array}
$$

In the spirit of Whitt's paper (1986) (Remark 2 p. 612) to compare $\varphi(\mathbf{X})$ and $\psi(\mathbf{Y})$ the Markov processes $(\varphi(\mathbf{X}), \mathbf{X})$ and $(\psi(\mathbf{Y}), \mathbf{Y})$ with values in $\mathbb{N} \times E$ are used and a stochastically monotone Markov process $\mathbf{Z}$ with values in $\mathbb{I N}$ is introduced in the middle. Then, the comparison conditions will appear as the conditions (16) and (17) uniformly in the extra information needed to pass from the non-Markov process $\varphi(\mathbf{X})$ to the Markov's one $(\varphi(\mathbf{X}), \mathbf{X})$. This appears clearly in conditions (18) 
and (19). But in our setting the use of a monotone process can be avoided.

Example 2. The stochastic comparison of systems with redundance are now completely studied. This example has been introduced by Cocozza and Rossignol (1995). Their comparison technique is very complicated and one condition (our condition 3)) is forgotten. We assert that the coupling technique developped here is easier to use and more reliable. Moreover, it gives the necessary and sufficient stochastic comparison conditions. The reason is that, in trying to construct a process staying in $\mathrm{K}$ and with the adequate marginal rates, the comparison conditions appear very clearly.

The systems (S) has two kinds of components:

* the components $\mathcal{C}_{D}=\left\{x_{1}, \cdots, x_{H}\right\}$ doubled by the components $\mathcal{C}_{S}=\left\{y_{1}, \cdots y_{H}\right\}$ in passive redundancy.

* the components $\mathcal{C}_{I}=\left\{c_{1}, \cdots, c_{K}\right\}$ without components in redundancy.

The set of all components is denoted by $\mathcal{C}=\mathcal{C}_{D} \cup \mathcal{C}_{S} \cup \mathcal{C}_{I}$. The generic pair $\{x, y\}$ must be understood as a component $x$ with its spare component. The state space of the system is $E \subset\{0,1\}^{2 H+K}$ where 1's stand for operational components. If the system is in state $\eta, \eta(x)(x \in \mathcal{C})$ is the state of component $x, \lambda(x, \eta)$ is the failure rate (resp., $\mu(x, \eta)$ the repair rate) of the component $x$ in the configuration $\eta$. For $x \in \mathcal{C}_{D}$, if $x$ is working, then $y$ is waiting and cannot fail. If $x$ fails, $y$ can refuse to start with probability $\gamma(y)$ and becomes immediately a failure component. The evolution of the system is described by a Markov Jump process $\mathbf{X}=\left\{X_{t}, t \geq 0\right\}$ with state space $E$. The jumps of $\mathbf{X}$ and their rates are deduced from the preceding description.

For $x \in \mathcal{C}_{I}$ and $i=0,1, b(\eta,\{x\}, i)$ is the rate of the jump from $\eta$ to $\eta^{x, i}$ where $\eta^{x, i}(x)=i$ and $\eta^{x, i}(y)=y \quad \forall y \neq x$. Then:

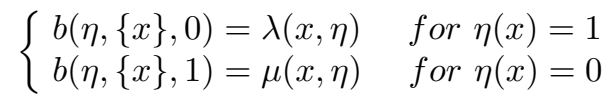

With a similar definition for the rate $b(\eta,\{x, y\},\{i, j\})$, we have : if $\eta(x)=1 \quad \eta(y)=1$

$$
\left\{\begin{array}{l}
b(\eta,\{x, y\},\{0,1\})=[1-\gamma(y)] \lambda(x, \eta) \\
b(\eta,\{x, y\},\{0,0\})=\gamma(y) \lambda(x, \eta)
\end{array}\right.
$$

if $\eta(x)=0 \quad \eta(y)=1$

$$
\left\{\begin{array}{l}
b(\eta,\{x, y\},\{1,1\})=\mu(x, \eta) \\
b(\eta,\{x, y\},\{0,0\})=\lambda(y, \eta)
\end{array}\right.
$$

if $\eta(x)=1 \quad \eta(y)=0$ (This state is reached after a repair of $x$ coming from $\zeta$ such that $\zeta(x)=0=\zeta(y))$. 


$$
\begin{gathered}
\left\{\begin{array}{l}
b(\eta,\{x, y\},\{1,1\})=\mu(y, \eta) \\
b(\eta,\{x, y\},\{0,0\})=\lambda(x, \eta) .
\end{array}\right. \\
\text { if } \eta(x)=0 \quad \eta(y)=0 \\
\left\{\begin{array}{l}
b(\eta,\{x, y\},\{1,0\})=\mu(x, \eta) \\
b(\eta,\{x, y\},\{0,1\})=\mu(y, \eta) .
\end{array}\right.
\end{gathered}
$$

All the other rates are null.

Remark. The repair rates for components in redundancy, can be treated alone, as the repair rates for components of $\mathcal{C}_{I}$.

The state space $E$ is endowed with the ordering induced by the natural ordering on $\{0,1\}^{2 H+K}$, coordinate by coordinate.

$$
\eta \leq \zeta \quad \eta \quad \eta(x) \leq \zeta(x) \quad \forall x \in \mathcal{C}
$$

In such a system any jump links two comparable configurations. They correspond to a degradation or a repair of the system.

Let (S) and (S') be two identical such systems, $\mathbf{X}=\left\{X_{t}, t \geq 0\right\}$ and

$\mathbf{Y}=\left\{Y_{t}, t \geq 0\right\}$ the corresponding Markov jump processes. As in the rest of this paper, the rates for $\mathbf{Y}$ are indexed with a prime. For commodity, the initial state is supposed to be the state for which all components are operational.

At first, necessary and sufficient conditions for $\stackrel{\mathcal{D}}{\leq}$ stochastic comparison of $\mathbf{X}$ and $\mathbf{Y}$ are given. They complete the sufficient conditions already obtained by Cocozza and Roussignol (1995 Prop. 5.3). One interest of our method, is perhaps, its simplicity. In trying to construct a process, staying in $K$ and with the adequate marginal rates, the comparison conditions appear very clearly.

Then, some natural functions of interest in such a system, are introduced, and the corresponding necessary and sufficient conditions for $\leq_{\varphi \psi}$ comparison conditions are given.

\section{Proposition $3 \quad \mathrm{X} \stackrel{\mathcal{D}}{\leq} \mathrm{Y}$}

if and only if $\forall(\eta, \zeta) \in E \times E$ s.t. $\eta \leq \zeta$

1) $\forall x \in \mathcal{C} \quad \mu(x, \eta) \leq \mu^{\prime}(x, \zeta)$ when $\eta(x)=0=\zeta(x)$.

2) $\forall x \in \mathcal{C} \quad \lambda(x, \eta) \geq \lambda^{\prime}(x, \zeta)$ when $\eta(x)=1=\zeta(x)$.

3) $\forall\{x, y\} \in \mathcal{C}_{D} \cup \mathcal{C}_{S}$

$\lambda(y, \eta) \geq \gamma^{\prime}(y) \lambda^{\prime}(x, \zeta)$ when $\eta(x)=0, \zeta(x)=1$ and $\eta(y)=1=\zeta(x)$.

4) $\forall\{x, y\} \in \mathcal{C}_{D} \cup \mathcal{C}_{S}$

$\gamma(y) \lambda(x, \eta) \geq \gamma^{\prime}(y) \lambda^{\prime}(x, \zeta)$ when $\eta(x)=1=\zeta(x)$ and $\eta(y)=1=\zeta(x)$.

The conditions 1), 2), 3) and 
5) $\forall y \in \mathcal{C}_{R} \quad \gamma(y) \geq \gamma^{\prime}(y)$

are sufficient to ensure $\mathbf{X} \stackrel{\mathcal{D}}{\leq} \mathbf{Y}$.

Proof. The technique already described in section 4) is used with

$$
K=\{(\eta, \zeta) \in E \times E \text { s.t. } \eta \leq \zeta\} .
$$

With some additional remarks, it allows a very simple and short proof.

Note at first, that hypothesis $\left(\mathbf{H}_{\mathbf{2}}\right)$ is satisfied here. Indeed, it is easy to show that it is always the case when $K$ is a comparison set and when the processes jump only between comparable states.

For $x \in \mathcal{C}_{I}$ the conditions 1) and 2) are the classical comparison conditions for birth and death processes. Now, consider a pair $\{x, y\}$ in $\mathcal{C}_{S} \cup \mathcal{C}_{D}$. A departure from $K$ by a jump of $\mathbf{X}$ is always a repair of a failed component : it can be compensated only by a repair of the same component for $\mathbf{Y}$ and the condition 1) is obtained.

Otherwise, all the cases are considered.

1) $(\eta(x), \eta(y)=(1,0)$ and $(\zeta(x), \zeta(y)=(1,0)$

The component $x$ (or $y$ ) must fail more quickly for (S). Then

$$
\lambda(x, \eta) \geq \lambda^{\prime}(x, \zeta) .
$$

2) $(\eta(x), \eta(y)=(0,1)$ and $(\zeta(x), \zeta(y)=(0,1))$

the same argument holds and the condition is:

$$
\lambda(y, \eta) \geq \lambda^{\prime}(y, \zeta) .
$$

3) $(\eta(x), \eta(y)=(1,0)$ and $(\zeta(x), \zeta(y)=(1,1)$

If $\mathbf{Y}$ jumps to $(0,0)$ or to $(0,1), \mathbf{X}$ must jump to $(0,0)$. Here $\left(\mathbf{H}_{\mathbf{1}}\right)$ is satisfied and the condition is:

$$
\lambda(x, \eta) \geq \gamma^{\prime}(y) \lambda^{\prime}(x, \zeta)+\left[1-\gamma^{\prime}(y)\right] \lambda^{\prime}(x, \zeta) .
$$

i.e.

$$
\lambda(x, \eta) \geq \lambda^{\prime}(x, \zeta) .
$$

4) $(\eta(x), \eta(y)=(0,1)$ and $(\zeta(x), \zeta(y)=(1,1)$

If $\mathbf{Y}$ jumps to $(0,0), \mathbf{X}$ must also jump to $(0,0)$ and the condition is

$$
\lambda(y, \eta) \geq \gamma^{\prime}(y) \lambda^{\prime}(x, \zeta) .
$$

5) $(\eta(x), \eta(y)=(1,1)$ and $(\zeta(x), \zeta(y)=(1,1)$

As in the preceding case, for the jump of $\mathbf{Y}$ to $(0,0)$, the first condition is

$$
\gamma(y) \lambda(x, \eta) \geq \gamma^{\prime}(y) \lambda^{\prime}(x, \zeta) .
$$

If $\mathbf{Y}$ jumps to $(0,1), \mathbf{X}$ can jump to $(0,0)$ with the residual rate 


$$
\gamma(y) \lambda(x, \eta)-\gamma^{\prime}(y) \lambda^{\prime}(x, \zeta)
$$

or to $(0,1)$ with rate $[1-\gamma(y)] \lambda(x, \eta)$. The condition is

$$
[1-\gamma(y)] \lambda(x, \eta)+\gamma(y) \lambda(x, \eta)-\gamma^{\prime}(y) \lambda^{\prime}(x, \zeta) \geq\left[1-\gamma^{\prime}(y)\right] \lambda^{\prime}(x, \zeta) .
$$

or, after simplification

$$
\lambda(x, \eta) \geq \lambda^{\prime}(x, \zeta) .
$$

Suppose now, that exceptional failures can occur to the system with rate $\Lambda(\eta)$. When such a general failure occurs, each operational component fails with probability $m(x, \eta)$. Then, the proper failure rate of each component $x \in \mathcal{C}$ is now

$$
\lambda^{\prime}(x, \eta)=\lambda(x, \eta)+\Lambda(\eta) m(x, \eta) \text { if } \eta(x)=1 .
$$

The necessary and sufficient conditions for $\stackrel{\mathcal{D}}{\leq}$ stochastic comparison are the same as in Proposition (3) with $\lambda^{\prime}(x, \eta)$ instead of $\lambda(x, \eta)$.

In particular, the conditions of Proposition (3) together with

$$
\forall(\eta, \zeta) \in E \times E \text { s.t. } \eta \leq \zeta
$$

7) $\Lambda(\eta) \geq \Lambda^{\prime}(\zeta)$

8) $\forall x \in \mathcal{C} \quad m(x, \eta) \geq m^{\prime}(x, \zeta)$ if $\eta(x)=\zeta(x)=1$

are sufficient to have $\mathbf{X} \stackrel{\mathcal{D}}{\leq} \mathbf{Y}$.

Suppose now that the systems (S) and (S') are stochastically compared through the number of operational components. That is the $\leq_{\varphi \psi}$ comparison with $\varphi=\psi$, the number of operational components of $(\mathrm{S})$ and (S'). Note that it is possible here to consider the same kind of systems but with different state spaces $E$ and $F$. From $n \in\{0, \cdots, 2 H+K\}$, the possible jumps of $\varphi(\mathbf{X})$ are $n-2, n-1$ or $n+1$ (excepted for $n=0,1$ or $2 H+K$ ). Before considering the rates, it appears immediately that the construction of the coupling process is almost always impossible. Indeed, suppose that $\varphi(\eta)=\varphi(\zeta)=n$ and (S') has still an operational pair $\{x, y\}$ but not (S). In this configuration, a double failure for the pair $\{x, y\}$ for (S') cannot be balanced by a similar failure for $(\mathrm{S})$. In terms of processes, in this state, it is impossible to prevent any coupling process from going out of $K$.

On the other hand, it is possible to compare both systems through the number of operational pairs $\{x, y\}$.

Let $\varphi=\psi$ be the number of operational pairs $\{x, y\}$

$$
\varphi \quad: \quad E \longrightarrow\{0, \cdots, H\} .
$$

Then $\varphi(\mathbf{X})$ has the jumps of a classical birth and death process. The following necessary and sufficient conditions of $\leq_{\varphi \psi}$ comparison are directly deduced from Proposition (2). 


\section{Proposition $4 \quad \mathrm{X} \leq_{\varphi \psi} \mathbf{Y}$}

if and only if

$$
\begin{aligned}
& \forall n \in\{0, \cdots, H\} \quad \forall(\eta, \zeta) \in E \times E \text { s.t. } \varphi(\eta)=\varphi(\zeta)=n \\
& \text { 1) } \sum_{x: \eta(x)=0 \eta(y)=1} \mu(x, \eta)+\sum_{x: \eta(x)=1} \sum_{(y)=0} \mu(y, \eta) \\
& \quad \sum_{x: \zeta(x)=0 \zeta(y)=1} \mu^{\prime}(x, \zeta)+\sum_{x: \zeta(x)=1 \zeta(y)=0} \mu^{\prime}(y, \eta) . \\
& \quad \sum_{x: \eta(x)=1 \eta(y)=1} \gamma(y) \lambda(x, \eta)+\sum_{x: \eta(x)=1 \eta(y)=0} \lambda(x, \eta)+\sum_{x: \eta(x)=0} \lambda(y)=1 \\
& \geq \sum_{x: \zeta(x)=1 \zeta(y)=1} \gamma^{\prime}(y) \lambda^{\prime}(x, \zeta)+\sum_{x: \zeta(x)=1 \zeta(y)=0} \lambda^{\prime}(x, \zeta)+\sum_{x: \zeta(x)=0 \zeta(y)=1} \lambda^{\prime}(x, \eta) .
\end{aligned}
$$

At last, consider the state function $\varphi=\left(\varphi_{1}, \varphi_{2}\right)=\psi$ where $\varphi_{1}$ is the number of operational pairs $\{x, y\}$ and $\varphi_{2}$ the number of operational components of $\mathcal{C}_{I}$. The process $\varphi(\mathbf{X})$ with state space $\mathbb{I N} \times \mathbb{I N}$ has jumps of +1 or -1 for each coordinate. With the coupling technique, it is possible to obtain the conditions of stochastic comparison for such birth and death process in $\mathbb{N} \times \mathbb{I N}$ (cf. Doisy (1992a)) and then, to deduce as in Proposition (2), the conditions for $\leq_{\varphi \psi}$ comparison.

\section{Conclusion}

The interest to have explicit conditions of $\leq_{\varphi \psi}$ stochastic comparison are developed in conclusion and some field of possible use are mentioned.

It is well known that the convergence in distribution and the $\stackrel{\mathcal{D}}{\leq}$ are compatible in the sense that

$$
\text { if } P_{n}^{1} \stackrel{\mathcal{D}}{\leq} P_{n}^{2} \forall n \text { and } P_{n}^{i} \Longrightarrow P^{i} \text { as } n \rightarrow \infty \text { for } i=1,2 \text { then } P^{1} \stackrel{\mathcal{D}}{\leq} P^{2} .
$$

The same is true for the $\leq_{\varphi \psi}$ stochastic comparison. For the systems (S) and (S') compared through the number of operational pairs $\{x, y\}$ (see Proposition (4)) define $\forall n \in\{0, \cdots, H-1\}$

$$
\alpha_{n}^{+}=\max _{\eta \text { s.t. } \varphi(\eta)=n}\left[\sum_{\eta(x)=0 \eta(y)=1} \mu(x, \eta)+\sum_{\eta(x)=1 \eta(y)=0} \mu(y, \eta)\right]
$$


and $\forall n \in\{1, \cdots, H\}$

$$
\begin{aligned}
& \beta_{n}^{+}=\min _{\eta \text { s.t. } \varphi(\eta)=n} \\
& \left(\sum_{\eta(x)=1 \eta(y)=1} \gamma(y) \lambda(x, \eta)+\sum_{\eta(x)=1 \eta(y)=0} \lambda(x, \eta)+\sum_{\eta(x)=0 \eta(y)=1} \lambda(x, \eta)\right)
\end{aligned}
$$

(and $\alpha_{n}^{-}$and $\beta_{n}^{-}$with min and max exchanged). Let $\mathbf{Z}^{+}$and $\mathbf{Z}^{-}$be two birth and death processes with state space $\{0, \cdots, H\}$ and transition rates $\alpha_{n}^{+}, \beta_{n}^{+}$and $\alpha_{n}^{-}, \beta_{n}^{-}$respectively. By Proposition (2)

$$
\mathbf{Z}^{-} \leq_{i \varphi} \mathbf{X} \leq_{\varphi i} \mathbf{Z}^{+}
$$

where $i$ is the identity mapping of $\mathbb{I N}$. If $\pi$ is the stationary distribution of the (irreducible) Markov jump process $\mathbf{X}$ and $p^{+}, p^{-}$the stationary distributions of $\mathbf{Z}^{+}$and $\mathbf{Z}^{-}$(easy to compute), then

$$
p^{-} \leq_{i \varphi} \pi \leq_{\varphi i d} p^{+} .
$$

For example if $A_{i}$ is the set of configurations having at least $i$ failed pairs, then:

$$
\sum_{k=i}^{H} p_{k}^{-} \leq \pi\left(A_{i}\right) \leq \sum_{k=i}^{H} p_{k}^{+} \quad \forall i=1, \cdots, H .
$$

In particular $p_{H}^{-} \leq \pi\left(A_{H}\right) \leq p_{H}^{+}$.

In reliability theory, increasing coupling are very useful to compare reliability of systems and mean time to failure. Suppose that the state space $G$ is the disjoint union of the set $\mathcal{O}$ of operational states and the set $\mathcal{F}$ of failure states. Generally, the ordering is such that $\mathcal{O}$ is increasing and $\mathcal{F}$ decreasing. The possible jumps link only comparable states (repair or failures) and the starting point is the fully operational state. Let $T$ be the hitting time of $\mathcal{F}$ for $(\mathrm{S})$ (resp., $T^{\prime}$ for $\left(\mathrm{S}^{\prime}\right)$ ) and define $R(t)=P(T \geq t) \quad \forall t \geq 0$ (resp., $R^{\prime}(t)=P\left(T^{\prime} \geq t\right) \quad \forall t \geq 0$ ).

If $\mathbf{X} \stackrel{\mathcal{D}}{\leq} \mathbf{Y}$ it is clear, by the existence of the increasing coupling, that:

$$
\begin{aligned}
R(t) & \leq R^{\prime}(t) \quad \forall t \geq 0 \\
\mathbb{E}(T) & \leq \mathbb{E}\left(T^{\prime}\right) .
\end{aligned}
$$

If $\mathbf{X}$ and $\mathbf{Y}$ are compared through $\varphi \psi$ with values in $G$ the same is true with

$$
T_{\varphi}=\inf \left\{t \geq 0 \text { s.t. } X_{t} \in \varphi^{-1}(\mathcal{F})\right\} .
$$

and $T_{\psi}$ defined similarly. For example it can be applied starting from the prevoius relation :

$$
\mathbf{Z}^{-} \leq_{i \varphi} \mathbf{X} \leq_{\varphi i} \mathbf{Z}^{+} .
$$


For a birth and death process, it is easy to compute the mean time to reach one state starting from another (Karlin and Taylor (1975) p. 146). Then, bounds can be obtained for $\mathbb{E}\left(T_{\varphi}\right)$. Finally it seems interesting to develop the use of stochastic comparison when one wants to increase the probability of rare events (for example the total failure of a system). Explicit conditions on transitions rates allow to determine what rates must be increased (or decreased) to reach more quickly this rare event. Especially, it can be an important tool in the use of Importance Sampling (Goyal 1992).

\section{References}

1. W.J. Anderson. Continuous-Time Markov Chains. An Applications-Oriented Approach, Springer-Verlag(1991).

2. E. Cinlar. Introduction to Stochastic processes, Prentice-Hall, Inc. (1975).

3. C. Cocozza-Thivent, M. Roussignol. Techniques de Couplage en Fiabilité. Annales de l'I.H.P. Probabilités et Statistiques. Vol 31 N 1 p.114-141 (1995).

4. M. Doisy. Comparaison de processus à valeurs dans $\mathbb{Z}^{d}$. Actes des Ilèmes Journées de Mathématiques Appliquées Saragosse-Pau. Publication de l'U.P.P.A. (1992a).

5. F. Forbes, O. François and B. Ycart. Stochastic Comparison for resource sharing models. Markov Proc. Related Fields. 2 (4), p.581-605 (1996).

6. A. Goyal, P. Shahabuddin, P. Heidelberger, V.F. Nicola and P. Glynn. A Unified Framework for Simulating Markovian Models of Highly Dependable Systems. IEEE Trans. on Comp. Vol. 41, N 1, p. 36-50 (1992).

7. T. Kamae, U. Krengel and L. O'Brien. Stochastic Inequalities on Partially Ordered Spaces, The Annals of Probability. Vol.5, N 6, p.899-912 (1977).

8. S. Karlin and H.M. Taylor. A First Course in Stochastic processes, 2nd ed. Academic Press, New York (1975).

9. T. Lindvall. Lectures on the Coupling Method, Wiley, New York (1992).

10. T.M. Liggett. Interacting Particle Systems, Springer-Verlag, New York (1985).

11. A.W. Marshall and I. Olkin. Inequalities: Theory of Majorization and its Applications, Academic Press, New York (1979).

12. W.A. Massey. Stochastic Orderings for Markov processes on Partially Ordered Spaces, Mathematics of Operations Research, Vol.12, N 2, p.350-367 (1987)

13. T. Murata. Petri Nets: Property, Analysis and Applications, Proceedings of the I.E.E.E. Vol.77, N 4, (1989).

14. D. Stoyan. Comparison Methods for Queues and Other Stochastic Models, Wiley, New York (1983).

15. D. Strassen. The existence of probability measures with given marginals, Ann. Math. Statist. 36, 423-439 (1965).

16. W. Whitt. Stochastic Comparison for Non-Markov processes, Mathematics of Operations Research, Vol. 11, N 4, p.608-618 (1986).

17. S.Y. Zhang. (to appear). Existence and application of optimal Markovian coupling with respect to nonnegative lower semi-continuous functions. Acta Mathematica Sinica.

18. Y.H. Zhang. (to appear). Sufficient and necessary conditions for stochastic comparability of jump processes. Acta Mathematica Sinica. 


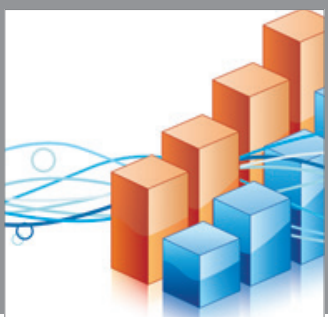

Advances in

Operations Research

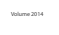

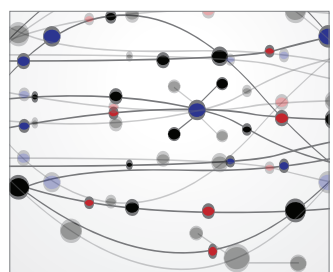

\section{The Scientific} World Journal
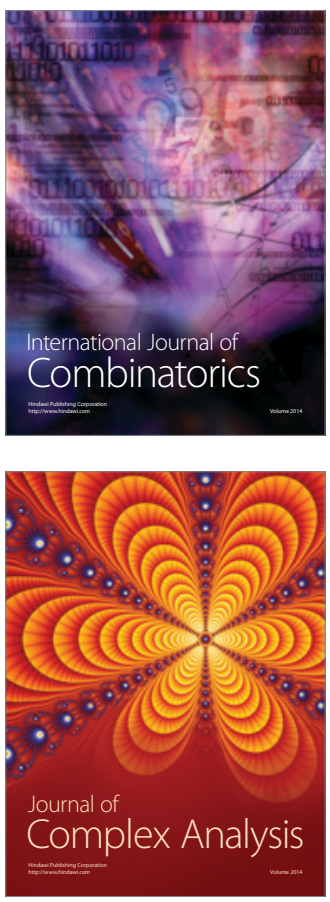

International Journal of

Mathematics and

Mathematical

Sciences
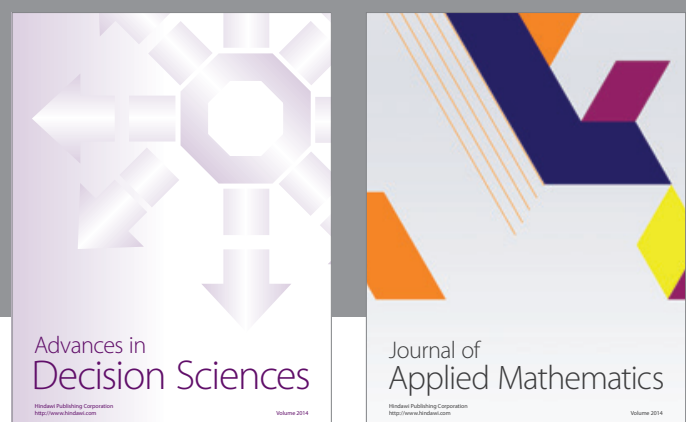

Journal of

Applied Mathematics
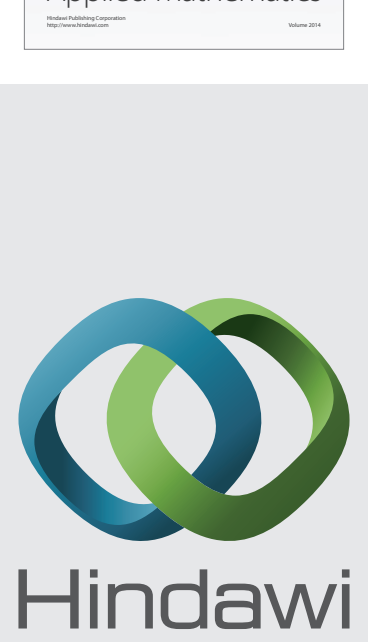

Submit your manuscripts at http://www.hindawi.com
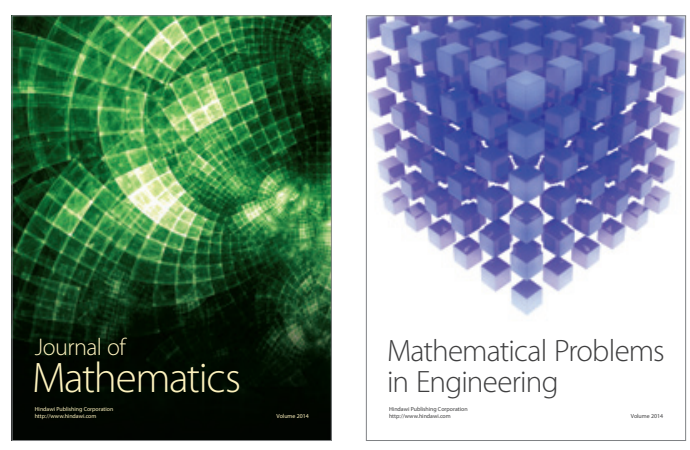

Mathematical Problems in Engineering
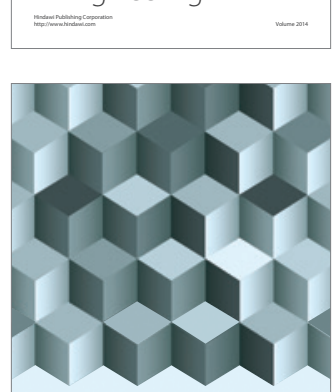

Journal of

Function Spaces
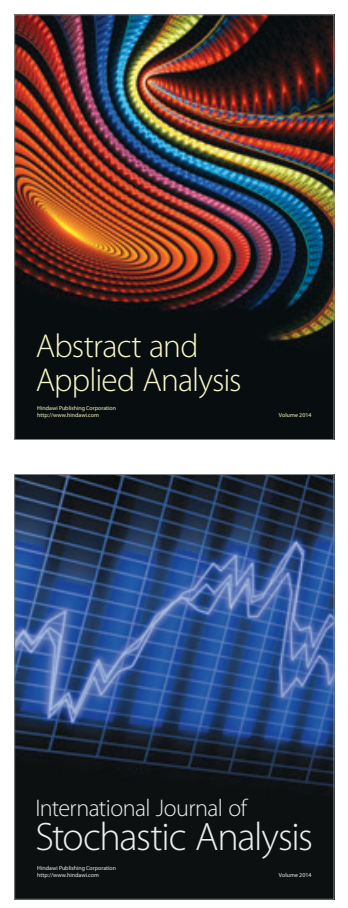

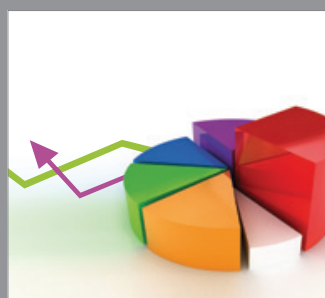

ournal of

Probability and Statistics

Promensencen
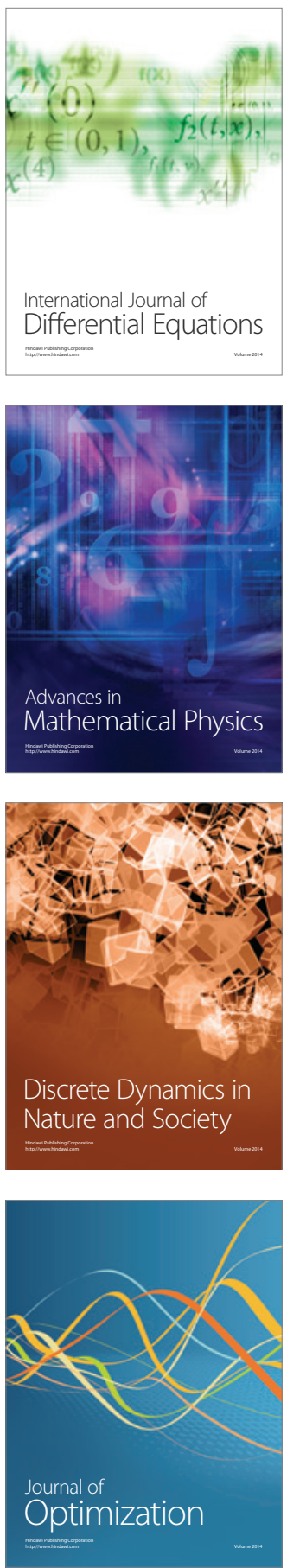